\title{
Uncovering the Detailed Structure of the Algebra Formed by the Invariant Charges of Closed Bosonic Strings Moving in $1+2$-Dimensional Minkowski Space
}

\author{
K. Pohlmeyer \\ Fakultät für Physik der Universität Freiburg, Hermann-Herder-Strasse 3, D-79104 Freiburg, Germany
}

Received: 10 June 1993

\begin{abstract}
For the Nambu-Goto theory of closed, bosonic strings moving in three space-time dimensions, generating relations of degree $\leq 6$ for the Poisson algebra of the infinitesimal generators of observable symmetry transformations have been computed. These computations result in a deeper insight into the structure of the symmetry algebra. In addition, a parametrization of the quantum corrections for the generating relations of degree 3 is given.
\end{abstract}

\section{Introduction}

By Wick rotation, the classical theory of relativistic bosonic closed strings moving in $(d-1)+1$-dimensional Minkowski space-time [1] is related to the geometry of complete minimal surfaces isometrically immersed in the $d$-dimensional Euclidean space [2]. With respect to a given (Cartesian) frame of reference, these surfaces and their immersions (apart from translations in some well-defined, surface-dependent direction) can be reconstructed from the knowledge of a certain infinite set of algebraically indepedent conformal invariants [3]. The reconstruction exploits the conformal structure of the minimal surfaces.

Along with previous investigations [3, 4, 6-10], the present analysis explores the intriguing possibility that the quantum theory of relativistic bosonic closed strings moving in $(d-1)+1$-dimensional Minkowski space-time is obtained from the geometry of complete minimal surfaces in the following way:

In a first step, the conformal invariants are turned into the (reparametrization invariant) elements of the algebra of infinitesimal observable symmetry generators ("invariant charges") of the classical string theory,

$$
\mathfrak{s o}(1, d-1)_{\text {boosts }} \uplus\left\{\mathbb{M}^{d} \oplus \mathfrak{h}_{\mathscr{P}}^{+} \oplus \mathfrak{h}_{: \mathfrak{p}}^{-}\right\} .
$$

Here the symbols $\mathbb{M}^{d}, \mathfrak{h}_{\mathscr{P}}^{+}$and $\mathfrak{h}_{\mathscr{P}}^{-}$stand for the linear span of the infinitesimal generators $\mathscr{P}_{\lambda}, \lambda=0,1, \ldots, d-1$, of rigid translations in Minkowski space, and

This work was supported by Volkswagenstiftung 
for the algebra of (translation invariant) "internal" invariant charges $\mathscr{Z}_{\mu_{1} \ldots \mu_{N}}^{ \pm(K)}$ for the left- and right-movers (with the components of the energy momentum vector being treated as $c$-numbers), respectively. In all cases, the field of coefficients is the field of complex numbers. The invariant charges $\mathscr{Z}_{\mu_{1} \ldots \mu_{N}}^{ \pm(K)}$ carry dimension (mass) ${ }^{N}$, degree of homogeneity $K$, "grade" $k=N-K$ and "degree" $\ell=N-K-1 . \mathfrak{h}_{\mathscr{P}}^{+}$and $\mathfrak{h}_{\mathscr{P}}^{-}$ form graded algebras, respectively, both under

i) the commutative ordinary multiplication, graded w.r.t. the grade $k$, and

ii) the non-commutative Poisson bracket operation, graded w.r.t. the degree $\ell$.

These algebras are presented in terms of their defining relations generating them from the commutative and associative ring of polynomials in the basis elements of the corresponding free Poisson algebras, i.e. generating them from the symmetric algebras over the free Lie algebras whose composition law is given by the Poisson bracket operation.

The observable content of the classical constraints is formulated in terms of the Casimir elements of the Poisson algebras $\mathfrak{h}_{\mathscr{p}}^{+}$and $\mathfrak{h}_{\mathscr{p}}^{-}$, respectively.

In a second step, "non-additive composition laws" for the invariant charges giving rise to a "Poisson bi-algebra structure" are specified. These laws compensate for the loss of analyticity in the transition from the theory of minimal surfaces to the theory of closed strings and include the classical "scattering" of closed strings.

In a third step, the algebras $\mathfrak{h}_{\mathscr{P}}^{+}$and $\mathfrak{h}_{\mathscr{P}}^{-}$are deformed, respectively, into algebras under associative multiplication and star operation, the Poisson bracket being replaced by $(i \hbar)^{-1}$ times the corresponding commutator, the complex conjugation being replaced by the star operation. The analogue goes for the respective bi-algebras, the non-additive composition laws being replaced by corresponding non-trivial comultiplication laws. The stated deformations should be subject to (an appropriate adaptation of) the requirement of coinciding Poincaré series and should correct the results of the Poisson bracket operation to the results of $(i \hbar)^{-1}$ times the corresponding commutation by polynomials in the (quantum version of the) invariant charges with coefficients exhibiting positive integer powers of $\hbar$. The quantum version of the classical constraints consists of a restriction of the values of the Casimir elements of the commutator algebras $\hat{\mathfrak{h}}_{\mathscr{P}}^{+}$and $\hat{\mathfrak{h}}_{\mathscr{P}}^{-}$.

In a fourth and final step, positive energetic, unitary, irreducible representations of the algebras $\hat{\mathfrak{h}}_{\tilde{p}}^{+}$and $\hat{\mathfrak{h}}_{\mathscr{P}}^{-}$as well as their respective bi-algebras are classified, representations which are subject to the constrains on the values of the Casimir elements.

There is a fair chance that this fiction is borne out by facts:

Firstly, it has been argued in ref. [4] that the renormalization of the invariant charges in WKB-approximation is attended with a single free parameter $\gamma$ only, this parameter being already familiar from the analysis of the anomalies of the free loop wave equation by Lüscher, Symanzik and Weisz [5], and that at least in WKBapproximation there does exist a representation of $\hat{\mathfrak{h}}_{\mathscr{P}}^{+} \oplus \hat{\mathfrak{h}}_{\mathscr{P}}^{-}$with a totally symmetric ground state.

Secondly, as the internal invariant charges commute with the components of the energy-momentum operator, and as in nature only finite energy-momentum degeneracies are observed, finite-dimensional representations of the algebras $\hat{\mathfrak{h}}_{\mathscr{2}}^{+}$and $\hat{\mathfrak{h}}_{-\infty}^{-}$meet predominant interest. For the existence of such representations, the algebras $\hat{\mathfrak{h}}_{\mathscr{P}}^{+}$and $\hat{\mathfrak{h}}_{\mathscr{P}}^{-}$must - and, in fact, do - possess ideals of finite co-dimension (see below). Since all representations of $\hat{\mathfrak{h}}_{\mathscr{P}}^{+} \oplus \hat{\mathfrak{h}}_{\mathscr{P}}^{-}$consists of entire multiplets of the little group 
(of the Lorentz group), any finite-dimensional representation of $\hat{\mathfrak{h}}_{\mathscr{P}}^{+} \oplus \hat{\mathfrak{h}}_{\bar{p}}^{-}$is bound to have a maximal spin-transfer. In any one of them, all invariant operator charges of sufficiently high spin must be represented by the zero operator, and, in addition, for the representation at hand there must exist a finite subset of invariant (operator) charges of lower spin such that any invariant (operator) charge can be expressed as a function of these.

Thirdly, as suggested by the combined behaviour of the grade $k$ under ordinary multiplication and the degree $\ell$ under Poisson bracket operation of the classical invariant charges $\mathscr{Z}_{\mu_{1} \ldots \mu_{N}}^{ \pm K}$, their quantum counterparts $\hat{\mathscr{Z}}_{\mu_{1} \ldots \mu_{N}}^{ \pm(K)}$ are uniformly representable as

$$
\hat{\mathscr{Z}}_{\mu_{1} \ldots \mu_{N}}^{ \pm(K)}=\left(\hbar /\left(2 \pi \alpha^{\prime}\right)\right)^{N-K}\left(\hat{\mathscr{P}}^{2}\right)^{K-\frac{N}{2}} \cdot \hat{\mathbb{Z}}_{\mu_{1} \ldots \mu_{N}}^{ \pm(K)}
$$

with the dimensionless operator $\hat{\bigotimes}_{\mu_{1} \ldots \mu_{N}}^{ \pm(K)}$ acting by discrete amounts and commuting with the mass operator $\hat{\mathscr{P}}^{2}$. Here, the symbol $\alpha^{\prime}$ denotes the inverse string tension. This implies that any reference to Planck's constant $h$ can be completely eliminated from the defining relations of the algebras $\hat{\mathfrak{h}}_{\mathscr{p}}^{+}$and $\hat{\mathfrak{h}}_{\text {p }}^{-}$.

Fourthly, maximal abelian subalgebras of the classical algebras $\mathfrak{h}_{\not p}^{+}$and $\mathfrak{h}_{\mathscr{p}}^{-}$for $\operatorname{Pp}^{2}=\mathfrak{m}^{2}>0$ have been identified [7] and so have been the Casimir elements of $\mathfrak{h}_{\mathscr{\rho}}^{+}$and $\mathfrak{h}_{\mathscr{\rho}}^{-}$[8]. Also, the observable content of the classical constraints has been formulated in terms of these Casimir elements.

Fifthly, the emergence of non-additive composition laws for the invariant charges has been noted in ref. [9].

Sixthly, for the special case $d=3$, there is some control over the derived algebras $\left\{\mathfrak{h}_{\text {p }}^{ \pm}, \mathfrak{h}^{ \pm}\right\}$, cp. ref. [10], and some of the relations defining the algebras $\mathfrak{h}_{\mathscr{p}}^{+}$and $\mathfrak{h}_{\mathscr{p}}^{-}$ have been computed explicitly, viz. all relations of degree $\ell=3$ and the following relations of degree $\ell=4$ : the pseudo-scalar relations, the only "spin" $=5$ relation and one of the "spin" $=3$ relations.

In the present communication, for $d=3$ and $\alpha^{\prime}=1 /(2 \pi)$, I shall spell out the remaining $\ell=4$ relations and report the essential features of all the $\ell=5$ relations and of two especially important $\ell=6$ relations which have been computed explicitly (and which are available on microfilm). These pieces of evidence bring the general structure of the algebras $\mathfrak{h}_{\mathscr{\rho}}^{+}$and $\mathfrak{h}_{\mathscr{\rho}}^{-}$to the fore. With the help of the above-mentioned requirements for the quantum deformations, I parametrize the quantum corrections of the defining $\ell=3$ relations.

\section{Simplifications, Conventions and Basic Facts}

The algebras $\mathfrak{h}_{\mathscr{\rho}}^{+}$and $\mathfrak{h}_{\tilde{\rho}}^{-}$, with the ordinary multiplication as the composition law, are isomorphic. As Lie algebras, with the Poisson bracket operation as the composition law, they differ by a global factor -1 for the structure constants. Thus, it suffices to analyze the algebra $\mathfrak{h}_{\mathfrak{p} p}^{+}$. Henceforth, the superscript + will be suppressed.

The focus of interest concerning the structure of the Poisson algebra $\mathfrak{h}_{p}$ and the commutator algebra $\hat{\mathfrak{h}}_{\mathscr{P}}$ lies in the case $\mathscr{P}^{2}=\mathfrak{m}^{2}>0$ and $\hat{\mathscr{P}}^{2}=\mathfrak{m}^{2} \cdot \mathbb{1}>0$, respectively. Due to Lorentz invariance, without further loss of generality the momentum rest frame

$$
\mathscr{P}_{\mu}=\mathfrak{m} \delta_{\mu, 0} / \hat{\mathscr{P}}_{\mu}=\mathfrak{m} \delta_{\mu, 0} \cdot \mathbb{1}
$$


will be chosen as the frame of reference. The algebras $\mathfrak{h}_{(\mathfrak{m}, 0,0)}$ and $\hat{\mathfrak{h}}_{(\mathfrak{m}, 0,0)}$ will be denoted by $\mathfrak{h}$ and $\hat{\mathfrak{h}}$, respectively.

The algebra $\mathfrak{h}$ is not finitely generated. However, in terms of certain iterated integrals, the generators are explicitly given [6]. For the case at hand, $d=3$, they are:

i) the infinitesimal generator of rotations in the $(1,2)$-plane:

$$
Q=\left(-\frac{1}{2} \mathfrak{m}^{-1}\right) \mathscr{Z}_{012}^{(2)}
$$

the degree of $Q$ being set equal to zero,

ii) the invariant charges of degree 1: $L_{s}$, "spin" $s= \pm 1, \pm 2$ with $\left\{Q, L_{2}\right\}=\frac{1}{i} s L_{s}$, and

iii) the (scalar) exceptional elements $L_{(1)}\left(=L_{0}\right), L_{(3)}, L_{(5)}, \ldots$ carrying degree 1,3 , $5, \ldots$, respectively:

$$
L_{(1)}=\frac{1 !}{4} \sum_{i=1}^{2} \mathscr{Z}_{0 i 02}^{(2)}, \quad L_{(3)}=\frac{3 !}{4} \sum_{i=1}^{2} \mathscr{Z}_{000 i 000 i}^{(4)}, \quad L_{(5)}=\frac{5 !}{4} \sum_{i=1}^{2} \mathscr{X}_{00000 i 00000 i}^{(6)},
$$

(Note that - compared to ref. [6] - the convention regarding the sign of the spin has been changed.)

The effect of parity transformation on the invariant charges is realized by replacing $Q / \hat{Q}$ by $-Q /-\hat{Q}$ and $L_{s} / \hat{L}_{s}$ by $L_{-s} / \hat{L}_{-s}$, respectively, and leaving the exceptional elements unchanged.

Complex conjugation (star operation) maps $L_{s} / \hat{L}_{s}$ into $L_{-s} / \hat{L}_{-s}$ and leaves $Q / \hat{Q}$ and the exceptional elements unchanged. The algebras $\mathfrak{h} / \hat{\mathfrak{h}}$ are described in terms of generating relations, i.e. (additional) relations imposed on the symmetric algebra over the free Poisson/the universal enveloping algebra of the free Lie algebra generated by the elements $Q / \hat{Q}, L_{s} / \hat{L}_{s}, s= \pm 1, \pm 2$, and $L_{(\ell)} / \hat{L}_{(\ell)}, \ell=1,3,5, \ldots$ The simplest of these generating relations assign to the element $Q / \hat{Q}$ the rôle of measuring the spin.

The next simplest of these generating relations can be stated as follows [7]: it is possible to set up explicitly a $1: 1$ correspondence between the exceptional elements $L_{(\ell)} \ell=1,3,5, \ldots$ and certain (real) scalar elements $B_{\ell}$ of degree $\ell$ which are algebraically independent of each other and which are in involution. (For further characteristic properties of the scalar elements $B_{\ell}$, see below.)

For the case at hand, $d=3=$ odd integer, the next simplest generating relations can be stated as follows [7]: for every even integer $\ell \geq 0$, there exists an essentially unique pseudo-scalar real element $B_{\ell}$, a dominant invariant charge of degree $\ell$, such that all these algebraically independent pseudo-scalar elements are in involution with each other and with the aforementioned scalar elements $B_{2 n+1}$. By "essentially unique" I mean: unique up to normalization and addition of a polynomial in the pseudo-scalar elements carrying degrees $<\ell$. The scalar and the pseudo-scalar elements under consideration generate the said maximal abelian subalgebra.

There are no further generating relations of degree $\ell=0,1$ and 2 .

In the following section I shall report further generating relations of degree $\geq 3$. A tolerably systematic description will be given - faute de mieux - in terms of 
i) $Q / \hat{Q}$,

ii) simple Poisson brackets/commutators, at least one of the scalar elements corresponding to the elements $B_{\ell} / \hat{B}_{\ell}, \ell=3,5,7, \ldots$ being among the arguments of each Poisson bracket/commutator, and

iii) the "Hall basis" elements of the free Poisson algebra/the free commutator algebra generated by the elements $L_{0} / \hat{L}_{0}, L_{s} / \hat{L}_{s}, s= \pm 1, \pm 2$.

For the construction of the Hall basis [11], I stipulate the following admissible ordering: let $u$ and $v$ be two "standard monomials". I set $u<v$, iff the (Hall) "degree" of $u$ is smaller than the (Hall) "degree" of $v$, or the (Hall) "degrees" of $u$ and $v$ are equal and the spin of $u$ is smaller than the spin of $v$, or the (Hall) "degrees" and spins of $u$ and $v$ are equal and the non-periodic cyclic word $i_{1} i_{2} \ldots i_{n}$ of index letters $i_{r} \in[-2, \ldots,+2], r=1, \ldots, n$, (Hall) bracketing of which gives rise to $u$, "precedes" the non-periodic cyclic word $j_{1} j_{2} \ldots j_{n}$ of index letters $j_{s} \in[-2, \ldots,+2], s=1, \ldots, n$, (Hall) bracketing of which gives rise to $v$. (The non-periodic cyclic word $i_{1} i_{2} \ldots i_{n}$ is said to precede the non-periodic cyclic word $j_{1} j_{2} \ldots j_{n}$ iff - read as integer numbers -

$$
\operatorname{MIN}_{p \in S_{n}} i_{p(1)} i_{p(2)} \ldots i_{p(n)}<\operatorname{MIN}_{q \in S_{n}} j_{q(1)} j_{q(2)} \ldots j_{q(n)}
$$

or

$$
\underset{p \in S_{n}}{\operatorname{MIN}} i_{p(1)} i_{p(2)} \ldots i_{p(n)}=\underset{q \in S_{n}}{\operatorname{MIN}} j_{q(1)} j_{q(2)} \ldots j_{q(n)}
$$

and

$$
\operatorname{MIN}_{p \in Z_{n}} i_{p(1)} i_{p(2)} \ldots i_{p(n)}<\operatorname{MIN}_{q \in Z_{n}} j_{q(1)} j_{q(2)} \ldots j_{q(n)},
$$

$S_{n}$ and $Z_{n}$ denoting the symmetric group and the cyclic group of $n$ objects, respectively.)

Multiple Poisson brackets of $L_{s_{\imath}}, s_{\imath}=0, \pm 1, \pm 2$, will be denoted by $\mathbb{L}_{\left(s_{1}, \ldots, s_{n}\right)}$. The symbols

$$
\mathbb{L}_{\left(s_{1}, \ldots, s_{a},\left(s_{a+1}, \ldots, s_{b}\right), s_{b+1}, \ldots, s_{c},\left(s_{c+1}, \ldots, s_{d}\right), \ldots\right)}
$$

stand for the multiple Poisson brackets of $L_{s_{1}}, \ldots, L_{s_{a}}$,

$$
\mathbb{L}_{\left(s_{a+1}, \ldots, s_{b}\right)}, L_{s_{b+1}}, \ldots, L_{s_{c}}, \mathbb{L}_{\left(s_{c+1}, \ldots, s_{d}\right)}, \ldots
$$

Their spin is:

$$
s\left(\mathbb{L}_{\left(s_{1}, \ldots, s_{a},\left(s_{a+1}, \ldots, s_{b}\right), s_{b+1}, \ldots, s_{c},\left(s_{c+1}, \ldots, s_{d}\right), \ldots\right)}\right)=\sum_{i=1}^{\cdots} s_{i} .
$$

A corresponding notation is used for multiple commutator brackets of $\hat{L}_{s_{\imath}}, s_{\imath}=0$, $\pm 1, \pm 2$.

I conclude this section by specifying the scalar elements $B_{\ell}$ for $\ell=1,3,5$ and the pseudo-scalar elements $B_{\ell}$ for $\ell=0,2,4$ :

$$
\begin{aligned}
B_{1}= & L_{(1)}, \\
B_{3}= & 16 L_{(3)}-8 L_{(1)}^{2}-\left(\mathbb{L}_{(1,-2,1)}+\mathbb{L}_{(-1,2,-1)}\right)+\frac{1}{2}\left(\mathbb{L}_{(0,-2,2)}+\mathbb{L}_{(0,2,-2)}\right) \\
& -2\left(\mathbb{L}_{(0,-1,1)}+\mathbb{L}_{(0,1,-1)}\right)-48 i Q \cdot \mathbb{L}_{(1,-1)}-8 L_{-2} \cdot L_{2}+8 L_{-1} \cdot L_{1}, \\
B_{5}= & (16)^{4} L_{(5)}-(16)^{4} L_{(1)} \cdot L_{(3)}+\frac{1}{3} \times(16)^{4} L_{(1)}^{3}
\end{aligned}
$$


plus a polynomial in $Q, L_{ \pm 1}, L_{ \pm 2}$, and in the Poisson brackets involving $L_{s}$, $s=0, \pm 1, \pm 2$ (see appendix);

$$
\begin{aligned}
B_{0}= & Q \\
B_{2}= & \left.i \mathbb{L}_{(2,-2)}-4 \mathbb{L}_{(1,-1)}\right), \\
B_{4}= & i\left[\left(\frac{1}{2} \mathbb{L}_{(2,-2,-2,2)}+\frac{32}{9} \mathbb{L}_{((2,1),(-1,-2))}+16 \mathbb{L}_{(1,-2,0,1)}+16 \mathbb{L}_{((1,0),(1,-2))}\right.\right. \\
& -16 \mathbb{L}_{(0,-2,1,1)}+16 \mathbb{L}_{(1,-1,-1,1)}-64 i Q \cdot \mathbb{L}_{(0,-2,2)}+64 i Q \cdot \mathbb{L}_{(1,-2,1)} \\
& +128 i Q \cdot \mathbb{L}_{(0,-1,1)}+64 L_{-2} \cdot \mathbb{L}_{(2,0)}-128 L_{-1} \cdot \mathbb{L}_{(2,-1)}-(16)^{2} L_{-1} \cdot \mathbb{L}_{(1,0)} \\
& \left.-10 \times(16)^{2} Q^{2} \cdot \mathbb{L}_{(1,-1)}+13 \times(16)^{2} i Q \cdot L_{-2} \cdot L_{2}-(16)^{3} i Q \cdot L_{-1} \cdot L_{1}\right) \\
& \left.-\left(L_{s} \leftrightarrow L_{-s}, s=0, \pm 1, \pm 2, Q \leftrightarrow-Q\right)\right] .
\end{aligned}
$$

\section{The Generating Relations of Degree 3 for the Algebra $\mathfrak{h}$}

Besides the explicitly stated relations and their implications, there are 10 independent generating relations of degree 3 , one for each of the possible non-vanishing spin values: $s= \pm 1, \pm 2, \pm 3, \pm 4, \pm 5$. It suffices to state the relations for the positive spin values. The relations for negative spin values can be obtained by complex conjugation or by parity transformation.

$$
\begin{aligned}
& s=5: \quad 0=\mathbb{L}_{(2,1,2)}, \\
& s=4: \quad 0=9 \mathbb{L}_{(2,0,2)}+4 \mathbb{L}_{(2,1,1)}+48 L_{2}^{2} \text {, } \\
& s=3: \quad 0=3 \mathbb{L}_{(2,-1,2)}+7 \mathbb{L}_{(2,0,1)}+8 \mathbb{L}_{(1,0,2)}+72\left(i Q \cdot \mathbb{L}_{(2,1)}-L_{1} \cdot L_{2}\right) \text {, } \\
& s=2: \quad 0=2 \mathbb{L}_{(2,-1,1)}+\mathbb{L}_{(1,-1,2)}+6 \mathbb{L}_{(1,0,1)}+24\left(i Q \cdot \mathbb{L}_{(2,0)}-2 L_{1}^{2}-8 Q^{2} \cdot L_{2}\right) \text {, } \\
& s=1: \quad 0=\mathbb{L}_{(2,-2,1)}+2 \mathbb{L}_{(1,-2,2)}-3 \mathbb{L}_{(2,-1,0)}+3 \mathbb{L}_{(0,-1,2)}-6 \mathbb{L}_{(1,-1,1)} \\
& -12 \mathbb{L}_{(1,0,0)}+24\left(i Q \cdot \mathbb{L}_{(2,-1)}-3 L_{-1} \cdot L_{2}+24 Q^{2} \cdot L_{1}\right) \text {. }
\end{aligned}
$$

Note the absence of factors $B_{1}\left(=L_{0}\right)$ from the right-hand sides of the above relations.

\section{The Generating Relations of Degree 4 for the Algebra $\mathfrak{h}$}

There exist elements of degree $\ell=4$ for all possible values of the spin between +5 and -5 . Besides the explicitly stated relations and their implications, there are 31 further independent generating relations: one for $s=5(-5)$, two for $s=4(-4)$, three for $s=3(-3)$, four for $s=2(-2)$, four for $s=1(-1)$ and three for $s=0$, among them one scalar relation. Again it suffices to state the generating relations for positive spin values:

$$
\begin{aligned}
s=5: \quad 0= & \mathbb{L}_{(1,0,2,2)}-\frac{2}{9} \mathbb{L}_{(2,1,1,1)}-\frac{16}{3} L_{2} \cdot \mathbb{L}_{(2,1)} ; \\
s=4: \quad 0= & \mathbb{L}_{(2,-2,2,2)}-24 \mathbb{L}_{(1,0,1,2)}+\frac{16}{3} \mathbb{L}_{((2,1),(1,0))}+\frac{2}{9} \times(16)^{2} i Q \cdot \mathbb{L}_{(2,1,1)} \\
& -128 L_{1} \cdot \mathbb{L}_{(2,1)}+128 L_{2} \cdot \mathbb{L}_{(2,0)}+\frac{41}{3} \times(16)^{2} i Q \cdot L_{2}^{2}, \\
0= & \mathbb{L}_{(2,0,1,1)}-10 \mathbb{L}_{(1,0,1,2)}+\frac{104}{3} i Q \cdot \mathbb{L}_{(2,1,1)}-96 L_{1} \cdot \mathbb{L}_{(2,1)} \\
& +48 L_{2} \cdot \mathbb{L}_{(2,0)}+104 \times 16 i Q \cdot L_{2}^{2} ;
\end{aligned}
$$




$$
\begin{aligned}
& s=3: \quad 0=3 \mathbb{L}_{(1,-2,2,2)}+4 \mathbb{L}_{(2,0,0,1)}-16 \mathbb{L}_{(1,0,0,2)}-10 \mathbb{L}_{((2,0),(1,0))} \\
& -12 \mathbb{L}_{(1,0,1,1)}+8 i Q \cdot \mathbb{L}_{(2,0,1)}-80 i Q \cdot \mathbb{L}_{(1,0,2)} \\
& -192 Q^{2} \cdot \mathbb{L}_{(2,1)}-96 L_{1} \cdot \mathbb{L}_{(2,0)}+192 L_{2} \cdot \mathbb{L}_{(2,-1)} \\
& +240 L_{2} \cdot \mathbb{L}_{(1,0)}+204 \times 16 i Q \cdot L_{1} \cdot L_{2} \text {, } \\
& 0=+9 \mathbb{L}_{(0,-1,2,2)}-4 \mathbb{L}_{((2,1),(1,-1))}+3 \mathbb{L}_{(2,0,0,1)}+24 \mathbb{L}_{(1,0,0,2)} \\
& +33 \mathbb{L}_{((2,0),(1,0))}+20 \mathbb{L}_{(1,0,1,1)}-144 i Q \cdot \mathbb{L}_{(2,0,1)} \\
& -120 i Q \cdot \mathbb{L}_{(1,0,2)}+960 Q^{2} \cdot \mathbb{L}_{(2,1)}+536 L_{1} \cdot \mathbb{L}_{(2,0)} \\
& -48 L_{2} \cdot \mathbb{L}_{(2,-1)}-376 L_{2} \cdot \mathbb{L}_{(1,0)}-228 \times 16 i Q \cdot L_{1} \cdot L_{2}, \\
& 0=3 \mathbb{L}_{(1,-1,1,2)}-\mathbb{L}_{((2,1),(1,-1))}-2 \mathbb{L}_{(1,0,1,1)}+40 L_{1} \cdot \mathbb{L}_{(2,0)} \\
& +64 L_{2} \cdot \mathbb{L}_{(1,0)}+108 \times 16 i Q \cdot L_{1} \cdot L_{2} \text {; } \\
& s=2: \quad 0=+\mathbb{L}_{(0,-2,2,2)}-2 \mathbb{L}_{((2,1),(1,-2))}+\frac{5}{2} \mathbb{L}_{(1,-1,0,2)}-13 \mathbb{L}_{((1,0),(2,-1))} \\
& +7 \mathbb{L}_{(0,-1,1,2)}-5 \mathbb{L}_{((2,0),(1,-1))}-9 \mathbb{L}_{((2,1),(0,-1))}-3 \mathbb{L}_{(2,0,0,0)} \\
& +13 \mathbb{L}_{(1,0,0,1)}-8 i Q \cdot \mathbb{L}_{(2,-2,2)}-24 i Q \cdot \mathbb{L}_{(1,-1,2)} \\
& +68 i Q \cdot \mathbb{L}_{(2,0,0)}+104 i Q \cdot \mathbb{L}_{(1,0,1)}-68 L_{-1} \cdot \mathbb{L}_{(2,1)} \\
& -320 Q^{2} \cdot \mathbb{L}_{(2,0)}+44 L_{1} \cdot \mathbb{L}_{(2,-1)} \\
& -328 L_{1} \cdot \mathbb{L}_{(1,0)}+16 L_{2} \cdot \mathbb{L}_{(2,-2)}-96 L_{2} \cdot \mathbb{L}_{(1,-1)} \\
& +96 i Q \cdot L_{1}^{2}+19 \times(16)^{2} i Q^{3} \cdot L_{2} \text {, } \\
& 0=2 \mathbb{L}_{(1,-2,1,2)}-\frac{2}{3} \mathbb{L}_{((2,1),(1,-2))}-\frac{7}{2} \mathbb{L}_{(1,-1,0,2)}+7 \mathbb{L}_{((1,0),(2,-1))} \\
& -\mathbb{L}_{(0,-1,1,2)}+2 \mathbb{L}_{((2,0),(1,-1))}+3 \mathbb{L}_{((2,1),(0,-1))}+3 \mathbb{L}_{(2,0,0,0)} \\
& -11 \mathbb{L}_{(1,0,0,1)}+16 i Q \cdot \mathbb{L}_{(1,-1,2)}-12 i Q \cdot \mathbb{L}_{(2,0,0)} \\
& -120 i Q \cdot \mathbb{L}_{(1,0,1)}+4 L_{-1} \cdot \mathbb{L}_{(2,1)}+192 Q^{2} \cdot \mathbb{L}_{(2,0)}+20 L_{1} \cdot \mathbb{L}_{(2,-1)} \\
& +264 L_{1} \cdot \mathbb{L}_{(1,0)}-16 L_{2} \cdot \mathbb{L}_{(2,-2)}+112 L_{2} \cdot \mathbb{L}_{(1,-1)} \\
& +42 \times 16 i Q \cdot L_{1}^{2}-9 \times(16)^{2} i Q^{3} \cdot L_{2}, \\
& 0=\mathbb{L}_{(1,-1,1,1)}+4 L_{-1} \cdot \mathbb{L}_{(2,1)}+4 L_{1} \cdot \mathbb{L}_{(2,-1)}+24 L_{1} \cdot \mathbb{L}_{(1,0)} \\
& -8 L_{2} \cdot \mathbb{L}_{(1,-1)}+288 i Q \cdot L_{1}^{2} \text {, } \\
& \left\{B_{3}, L_{2}\right\}=2 \mathbb{L}_{((2,1),(1,-2))}-\frac{15}{2} \mathbb{L}_{(1,-1,0,2)}+15 \mathbb{L}_{((1,0),(2,-1))} \\
& -9 \mathbb{L}_{(0,-1,1,2)}+3 \mathbb{L}_{((2,0),(1,-1))}+11 \mathbb{L}_{((2,1),(0,-1))} \\
& +5 \mathbb{L}_{(2,0,0,0)}-15 \mathbb{L}_{(1,0,0,1)}+12 i Q \cdot \mathbb{L}_{(2,-2,2)} \\
& +24 i Q \cdot \mathbb{L}_{(1,-1,2)}-60 i Q \cdot \mathbb{L}_{(2,0,0)}-120 i Q \cdot \mathbb{L}_{(1,0,1)} \\
& +108 L_{-1} \cdot \mathbb{L}_{(2,1)}-192 Q^{2} \cdot \mathbb{L}_{(2,0)}-36 L_{1} \cdot \mathbb{L}_{(2,-1)} \\
& +408 L_{1} \cdot \mathbb{L}_{(1,0)}-24 L_{2} \cdot \mathbb{L}_{(2,-2)}+96 L_{2} \cdot \mathbb{L}_{(1,-1)} \\
& -288 i Q \cdot L_{1}^{2}-37 \times(16)^{2} i Q^{3} \cdot L_{2} \text {; }
\end{aligned}
$$




$$
\begin{aligned}
& s=1: \quad 0=\mathbb{L}_{(0,-2,1,2)}-\frac{5}{3} \mathbb{L}_{(1,-2,1,1)}-\frac{3}{2} \mathbb{L}_{(1,-1,-1,2)}-\frac{3}{2} \mathbb{L}_{((2,-1),(1,-1))} \\
& -7 \mathbb{L}_{(1,-1,0,1)}+3 \mathbb{L}_{((1,0),(1,-1))}-3 \mathbb{L}_{(0,-1,1,1)}-8 i Q \cdot \mathbb{L}_{(1,-2,2)} \\
& -8 i Q \cdot \mathbb{L}_{(2,-1,0)}+8 i Q \cdot \mathbb{L}_{(0,-1,2)}-40 i Q \cdot \mathbb{L}_{(1,-1,1)} \\
& -\frac{40}{3} L_{-2} \cdot \mathbb{L}_{(2,1)}+12 L_{-1} \cdot \mathbb{L}_{(2,0)}+(16)^{2} Q^{2} \cdot \mathbb{L}_{(2,-1)} \\
& +2 \times(16)^{2} Q^{2} \cdot \mathbb{L}_{(1,0)}-\frac{56}{3} L_{1} \cdot \mathbb{L}_{(2,-2)} \\
& -48 L_{1} \cdot \mathbb{L}_{(1,-1)}+\frac{112}{3} L_{2} \cdot \mathbb{L}_{(1,-2)} \\
& +96 L_{2} \cdot \mathbb{L}_{(0,-1)}+62 \times 16 i Q \cdot L_{-1} \cdot L_{2}-(16)^{2} i Q^{3} \cdot L_{1}, \\
& 0=\mathbb{L}_{((2,0),(1,-2))}-\frac{4}{3} \mathbb{L}_{(1,-2,1,1)}-\mathbb{L}_{(2,-1,0,0)}+\mathbb{L}_{(0,-1,0,2)} \\
& -2 \mathbb{L}_{(1,-1,0,1)}+2 \mathbb{L}_{((1,0),(1,-1))}-2 \mathbb{L}_{(0,-1,1,1)}-8 i Q \cdot \mathbb{L}_{(1,-2,2)} \\
& -20 i Q \cdot \mathbb{L}_{(2,-1,0)}+4 i Q \cdot \mathbb{L}_{(0,-1,2)}-24 i Q \cdot \mathbb{L}_{(1,-1,1)} \\
& -\frac{32}{3} L_{-2} \cdot \mathbb{L}_{(2,1)}+24 L_{-1} \cdot \mathbb{L}_{(2,0)}+160 Q^{2} \cdot \mathbb{L}_{(2,-1)} \\
& +(16)^{2} Q^{2} \cdot \mathbb{L}_{(1,0)}-\frac{16}{3} L_{1} \cdot \mathbb{L}_{(2,-2)}-48 L_{1} \cdot \mathbb{L}_{(1,-1)} \\
& +\frac{56}{3} L_{2} \cdot \mathbb{L}_{(1,-2)}+16 L_{2} \cdot \mathbb{L}_{(0,-1)} \\
& +160 i Q \cdot L_{-1} \cdot L_{2}-(16)^{2} i Q^{3} \cdot L_{1} \text {, } \\
& 0=\mathbb{L}_{((2,1),(0,-2))}-\mathbb{L}_{(1,-2,1,1)}-\frac{3}{2} \mathbb{L}_{(1,-1,-1,2)}-\frac{3}{2} \mathbb{L}_{((2,-1),(1,-1))} \\
& -3 \mathbb{L}_{(1,-1,0,1)}+3 \mathbb{L}_{((1,0),(1,-1))}-3 \mathbb{L}_{(0,-1,1,1)} \\
& -24 i Q \cdot \mathbb{L}_{(1,-2,2)}+24 i Q \cdot \mathbb{L}_{(2,-1,0)}-24 i Q \cdot \mathbb{L}_{(0,-1,2)} \\
& +24 i Q \cdot \mathbb{L}_{(1,-1,1)}+96 i Q \cdot \mathbb{L}_{(1,0,0)}-8 L_{-2} \cdot \mathbb{L}_{(2,1)} \\
& +12 L_{-1} \cdot \mathbb{L}_{(2,0)}+384 Q^{2} \cdot \mathbb{L}_{(2,-1)}+384 Q^{2} \cdot \mathbb{L}_{(1,0)} \\
& -16 L_{1} \cdot \mathbb{L}_{(2,-2)}-48 L_{1} \cdot \mathbb{L}_{(1,-1)}+32 L_{2} \cdot \mathbb{L}_{(1,-2)} \\
& +96 L_{2} \cdot \mathbb{L}_{(0,-1)}+102 \times 16 i Q \cdot L_{-1} \cdot L_{2} \\
& -21 \times(16)^{2} i Q^{3} \cdot L_{1} \text {, } \\
& \left\{B_{3}, L_{1}\right\}=\frac{1}{4} \mathbb{L}_{(-1,-2,2,2)}-\frac{1}{2} \mathbb{L}_{(1,-2,1,1)}-\frac{9}{4} \mathbb{L}_{(2,-1,0,0)} \\
& +\frac{9}{4} \mathbb{L}_{(0,-1,0,2)}-\frac{3}{2} \mathbb{L}_{((2,0),(0,-1))}+\frac{3}{2} \mathbb{L}_{(1,-1,0,1)} \\
& -\frac{3}{2} \mathbb{L}_{((1,0),(1,-1))}-\frac{3}{2} \mathbb{L}_{(0,-1,1,1)}-4 \mathbb{L}_{(1,0,0,0)} \\
& -12 i Q \cdot \mathbb{L}_{(1,-2,2)}+15 i Q \cdot \mathbb{L}_{(2,-1,0)}-15 i Q \cdot \mathbb{L}_{(0,-1,2)} \\
& +18 i Q \cdot \mathbb{L}_{(1,-1,1)}+96 i Q \cdot \mathbb{L}_{(1,0,0)}+8 L_{-2} \cdot \mathbb{L}_{(2,1)} \\
& +6 L_{-1} \cdot \mathbb{L}_{(2,0)}+168 Q^{2} \cdot \mathbb{L}_{(2,-1)}-192 Q^{2} \cdot \mathbb{L}_{(1,0)} \\
& +16 L_{1} \cdot \mathbb{L}_{(2,-2)}-36 L_{1} \cdot \mathbb{L}_{(1,-1)}+4 L_{2} \cdot \mathbb{L}_{(1,-2)} \\
& +456 i Q \cdot L_{-1} \cdot L_{2}-308 \times 16 i Q^{3} \cdot L_{1} \text {; }
\end{aligned}
$$

$s=0$, scalar:

$$
\begin{aligned}
0= & \mathbb{L}_{((1,-1),(2,-2))}+\left(\mathbb{L}_{(0,-2,0,2)}+\mathbb{L}_{(0,2,0,-2)}\right) \\
& +4\left(\mathbb{L}_{((1,0),(1,-2))}+\mathbb{L}_{((-1,0),(-1,2))}\right)-\left(\mathbb{L}_{(0,-2,1,1)}+\mathbb{L}_{(0,2,-1,-1)}\right) \\
& +8 i Q \cdot\left(\mathbb{L}_{(1,-2,1)}-\mathbb{L}_{(-1,2,-1)}\right) \\
& -32 i Q \cdot \mathbb{L}_{(1,-1,0)}-32\left(L_{-2} \cdot \mathbb{L}_{(2,0)}+L_{2} \cdot \mathbb{L}_{(-2,0)}\right),
\end{aligned}
$$


$s=0$, pseudo-scalar:

$$
\begin{array}{rl}
0= & 3 \mathbb{L}_{((2,-1),(1,-2))}-\frac{1}{3} \mathbb{L}_{((2,1),(-1,-2))} \\
& +6\left(\mathbb{L}_{((1,0),(1,-2))}-\mathbb{L}_{((-1,0),(-1,2))}\right)-\left(\mathbb{L}_{(0,-2,1,1)}-\mathbb{L}_{(0,2,-1,-1)}\right) \\
& +2\left(\mathbb{L}_{(1,-1,-1,1)}-\mathbb{L}_{(-1,1,1,-1}\right)+12 \mathbb{L}_{((1,0),(0,-1))} \\
& +16\left[2 i Q \cdot\left(\mathbb{L}_{(1,-2,1)}+\mathbb{L}_{(-1,2,-1)}\right)+3 i Q \cdot\left(\mathbb{L}_{(0,-1,1)}+\mathbb{L}_{(0,1,-1)}\right)\right. \\
& -\left(L_{-2} \cdot \mathbb{L}_{(2,0)}-L_{2} \cdot \mathbb{L}_{(-2,0)}\right)+3\left(L_{-1} \cdot \mathbb{L}_{(2,-1)}-L_{1} \cdot \mathbb{L}_{(-2,1)}\right) \\
& +2\left(L_{-1} \cdot \mathbb{L}_{(1,0)}-L_{1} \cdot \mathbb{L}_{(-1,0}\right)-64 Q^{2} \cdot \mathbb{L}_{(1,-1)} \\
& \left.-16 i Q \cdot L_{-2} \cdot L_{2}-128 i Q \cdot L_{-1} \cdot L_{1}+(16)^{2} i Q^{5}\right] \\
0 & 3 \mathbb{L}_{((2,0),(0,-2))}-2\left(\mathbb{L}_{(0,-2,1,1)}-\mathbb{L}_{(0,2,-1,-1)}\right)-2\left(\mathbb{L}_{(1,-1,-1,1)}-\mathbb{L}_{(-1,1,1,-1)}\right) \\
& -24 i Q \cdot\left(\mathbb{L}_{(0,-2,2)}+\mathbb{L}_{(0,2,-2)}\right)+16 i Q \cdot\left(\mathbb{L}_{(1,-2,1)}+\mathbb{L}_{(-1,2,-1)}\right) \\
& +16\left(L_{-2} \cdot \mathbb{L}_{(2,0)}-L_{2} \cdot \mathbb{L}_{(-2,0}\right)+64\left(L_{-1} \cdot \mathbb{L}_{(1,0)}-L_{1} \cdot \mathbb{L}_{(-1,0)}\right) \\
& -192 Q^{2} \cdot \mathbb{L}_{(2,-2)}+(16)^{2} Q^{2} \cdot \mathbb{L}_{(1,-1)}+(16)^{2} i Q \cdot L_{-2} \cdot L_{2} \\
& +2 \times(16)^{2} i Q \cdot L_{-1} \cdot L_{1}-(16)^{3} i Q^{5} .
\end{array}
$$

Note the absence of factors $B_{1}\left(=L_{0}\right)$ and $B_{3}$ from the right-hand sides of the above relations.

In particular, I conclude from the last of the $s=2$ and $s=1$ relations that the Poisson bracket action of the scalar element $B_{3}$ (which has replaced the exceptional element $L_{(3)}$ ) maps the quotient of the symmetric algebra over the free Poisson algebra generated by $Q, L_{s}$, and $\left\{\ldots\left\{L_{s}, L_{0}\right\}, \ldots, L_{0}\right\}, s= \pm 1, \pm 2$, by the ideal generated by the remaining relations of degree $\leq 4$ linearly into itself.

\section{The Generating Relations of Degree 5 for the Algebra $\mathfrak{h}$}

There exist elements of degree $\ell=5$ for all possible values of the spin between $s=+6$ and -6 . Besides the explicitly stated relations and their implications, there are 17 further independent generating relations - a remarkably moderate increase in the number of generating relations: one for $s=4(-4)$, two for $s=3(-3)$, two for $s=2(-2)$, three for $s=1(-1)$, and one for $s=0$, the latter being a scalar relation. The relations are available on microfilm and will not be spelled out in detail here. Their most important feature consists again of the absence of (a priori possible) factors $B_{1}$ and/or $B_{3}$ in the polynomially non-linear parts of the relations among the multiple Poisson brackets of degree 5 involving the elements $L_{0}$ and $L_{s}, s= \pm 1, \pm 2$. Here, the generating relations of lower degree have been used. To wit, these non-linear parts of the generating relations are polynomials in $Q, L_{s}, s= \pm 1 . \pm 2$, and the simple or multiple Poisson brackets of degree $\leq 4$ with arguments $L_{0}, L_{s}, s= \pm 1, \pm 2$. This corroborates further the conjecture made in ref. [6] as to the generality of this feature for all generating relations of arbitrary degree. If this conjecture is indeed correct, then the set $U$ of all polynomials in $Q, L_{s}, s= \pm 1, \pm 2$, and in the simple or multiple Poisson brackets of arbitrary degree with arguments $L_{0}, L_{s}, s= \pm 1, \pm 2$, closes under Poisson bracket operation (and ordinary multiplication), i.e. forms an algebra both under Poisson bracket operation and ordinary multiplication. Moreover, the Poisson bracket actions of both $B_{1}\left(=L_{0}\right)$ and $B_{3}$ map the set $U$ linearly into itself. 
The set $U$ is quotient algebra of a subalgebra of the symmetric algebra over the free Poisson algebra generated by the finitely many elements $Q, L_{0}, L_{s}, s= \pm 1, \pm 2$, The double rôle played by the scalar element $B_{1}\left(=L_{0}\right)$ is a peculiarity of $2+1$ dimensional space-time. This irregularity gives way to a perfectly "democractic" scenario in $3+1$-dimensions.

Returning to the case at hand: $2+1$-dimensional space-time, it seems worth pointing out that the pseudo-scalar elements $B_{0}, B_{2}, B_{4}, \ldots$ generate a maximal abelian subalgebra of the algebra $U$ and - if the scalar element $L_{0}$ is added - a maximal abelian subalgebra of the said symmetric algebra.

\section{The Generating Relations of Degree 6 for the Algebra $h$ and the Apparent Structure of the Poisson Algebra of Observable Symmetries}

Time and again checked by the computer, it was possible to calculate the Poisson bracket action of the scalar element $B_{5}$ on the generators $L_{s}, s= \pm 1, \pm 2$. The formulae for the corresponding generating relations are available on microfilm, too. It turns out that the images of $L_{s}$ under the Poisson bracket action of $B_{5}$ are contained in the set $U$. This implies that $B_{5}$ maps the set $U$ linearly into itself.

It is now a fairly obvious guess that the algebra $\mathfrak{h}$ of internal invariant charges has the following structure: $\mathfrak{h}$ is the symmetric algebra over

$$
\mathfrak{A} \uplus U
$$

with $\mathfrak{A}$ the infinite dimensional abelian Poisson Lie algebra generated by $B_{\ell}$, $\ell=1,3,5,7, \ldots$ This amounts to a considerable specification and simplification of the structure of the algebra formed by the infinitesimal generators of observable symmetry transformations:

$$
\mathfrak{s o}(1,2)_{\text {boosts }} \uplus\left\{\mathbb{M}^{3} \oplus\left(\mathfrak{A}^{+} \uplus U^{+}\right) \oplus\left(\mathfrak{A}^{-} \uplus U^{-}\right)\right\}
$$

exhibiting, in particular, infinitely many ideals of finite co-dimension. This structure is expected to survive quantization.

\section{The Generating Relations of Order $\hbar^{4}$ for the Algebra $\hat{\mathfrak{h}}$ : Parametrization of the Quantum Corrections}

The inverse string tension $\alpha^{\prime}$ is reintroduced. I postulate the following properties of the quantum deformation of the Poisson algebra $\mathfrak{h}$ into the commutator algebra $\hat{\mathfrak{h}}$ :

i) There exists a $1: 1$ correspondence between the generators of the algebra $\mathfrak{h}: Q$, $L_{s}, s= \pm 1, \pm 2, L_{\emptyset)}, \ell=1,3,5, \ldots$ and algebraically independent elements of the algebra $\hat{\mathfrak{h}}: \hat{Q}, \hat{L}_{s}, s= \pm 1, \pm 2, \hat{L}_{\emptyset}, \ell=1,3,5, \ldots$ which in their turn generate the algebra $\hat{\mathfrak{h}}$. Each one of these generators of the algebra $\hat{\mathfrak{h}}$ carries a "weight" - with the interpretation: power in $\hbar /\left(\mathfrak{m}^{2} 2 \pi \alpha^{\prime}\right)$ - which is given by the grade of its classical counterpart.

ii) Consider the algebra $\hat{\mathfrak{h}}$ as a quotient of an auxiliary quotient of the free associative ring of polynomials in the generators $\hat{Q}, \hat{L}_{s}, s= \pm 1, \pm 2$, and $\hat{L}_{(1)}, \hat{L}_{(3)}, \hat{L}_{(5)}, \ldots$ identifying the Lie bracket with the commutator, i.e. of the universal enveloping algebra generated by $\hat{Q}, \hat{L}_{s}, s= \pm 1, \pm 2$, and $\hat{L}_{(1)}, \hat{L}_{(3)}, \hat{L}_{(5)}, \ldots$ The auxiliary 
quotient is to be formed w.r.t: the ideal defined by the generators: $\left[\hat{Q}, \hat{L}_{s}\right]-\hbar s \hat{L}_{s}$, and $\left[\hat{Q}, \hat{L}_{(\ell)}\right], s= \pm 1, \pm 2 ; \ell=1,3,5, \ldots$ Extend the above Hall basis of the free Poisson algebra/free commutator algebra to include the exceptional generators $L_{(\ell)} / \hat{L}_{\emptyset}, \ell=1,3,5, \ldots$ With the help of this extended Hall basis construct a basis for the auxiliary quotient algebra. The resulting basis elements consist of "words" with "letters" from this Hall basis in some fixed order.

Divide a given Hall basis element by $(i \hbar)$ to the power: number of commutations involved in the expression for the given basis element. Call the outcome the "adjusted" Hall basis element associated with the originally given basis element. Assign to the adjusted Hall basis element the weight: sum of the weights of the individual generators involved in the expression for the given basis element minus the number of commutations involved. The "adjusted words" with adjusted Hall basis elements as letters appearing in some fixed order still form a basis of the auxiliary quotient algebra. Assign to each such adjusted word the weight: sum of the weights of its individual letters.

iii) The generating relations of degree $\ell$ of the Poisson algebra $\mathfrak{h}$ can be decomposed according to their behaviour under rotations and parity transformation, i.e. they can be labelled - among other things - by their spin (and parity). Within the symmetry algebra over the free Poisson algebra, they express certain linear combinations of Hall basis elements of the free Poisson algebra, all of which carry the same degree $\ell$, spin $s$ (and parity), in terms of polynomials in $Q$ and in the Hall basis elements of the free Poisson algebra carrying lesser degree, the polynomials having neither a constant nor a linear part and not involving the exceptional generators as letters in their words. The commutator algebra $\hat{\mathfrak{h}}$ is obtained from the above auxiliary quotient of the free associative ring of polynomials by a set of quantum generating relations which are in a $1: 1$ correspondence to the classical generating relations. To each one of the classical generating relations of degree $\ell$, spin $s$ (and even or odd parity) there corresponds a quantum generating relation of order $\hbar^{\ell+1} \operatorname{spin} s$ (and even or odd parity). The quantum generating relation corresponding to a given classical generating relation of degree $\ell$, spin $s$ (and even or odd parity) expresses a definite linear combination of adjusted Hall basis elements in the quantum generators $\hat{Q}$, $\hat{L}_{s}, s= \pm 1, \pm 2, \hat{L}_{(1)}, \hat{L}_{(3)}, \hat{L}_{(5)}, \ldots$ - each one of the adjusted Hall basis elements carrying weight $(\ell+1)$, spin $s$ (the corresponding parities being either all even or all odd) - in terms of a polynomial in the adjusted Hall basis elements, such that the weight of each one of the adjusted words constituting the polynomial is equal to or smaller than $(\ell+1)$. The definite linear combination of adjusted Hall basis elements in the quantum generators just alluded to is obtained from the linear combination of Hall basis elements in the classical generators appearing in the corresponding classical generating relation by replacing the classical generators by their quantum counterparts and by replacing each Poisson bracket by the corresponding commutator divided by $(i \hbar)$. The polynomial alluded to above is not completely determined by the corresponding classical generating relation, as yet. However, it is required to have the following properties: (1) Including the coefficients into the counting, each term of the polynomial is of order $\hbar^{\ell+1}$. (2) That part of the polynomial which is made up of words, each one carrying weight equal to $(\ell+1)$ can be interpreted as a quantum ordering of the corresponding classical polynomial (carrying degree $\ell$ ) in the Hall basis elements in the classical generators of lesser degree. (3) Each term of the polynomial carries the same spin $s$. (4) The behaviour of the polynomial under parity transformation and star operation is given by the respective behaviour of the linear 
combination of adjusted commutators. (5) The requirement of consistency among the generating relations does not introduce any independent new relation which is not the uniquely defined counterpart of a classical relation. (6) The algebra $\hat{\mathfrak{h}}$ is the enveloping algebra of

with $\hat{\mathfrak{A}}$ an infinite dimensional abelian commutator algebra generated by hermitian scalar elements $\hat{B}_{\ell}, \ell=1,3,5, \ldots$, and $\hat{U}$ a commutator algebra generated by $\hat{Q}$, $\hat{L}_{s}$, and $\left[\ldots\left[\hat{L}_{s}, \hat{L}_{0}\right], \ldots, \hat{L}_{0}\right], s= \pm 1, \pm 2$. (Further restrictions on the polynomial under consideration are expected to result from the postulated bi-algebra structure.)

Applying the above postulates concerning the quantum deformation and employing an obvious notation, I arrive at the following description of the generating relations of order $\hbar^{4}$ in terms of 12 real parameters $a, b, \ldots, g, r, s, \ldots, v$ :

$$
\begin{aligned}
& s=5: \quad 0=-\frac{1}{\hbar^{2}} \hat{\mathbb{L}}_{[2,1,2]}, \\
& s=4: \quad 0=-\frac{9}{\hbar^{2}} \hat{\mathbb{L}}_{[2,0,2]}-\frac{4}{\hbar^{2}} \hat{\mathbb{L}}_{[2,1,1]}+\frac{48}{\left(2 \pi \alpha^{\prime}\right)^{2}} \hat{L}_{2}^{2}, \\
& s=3: \quad 0=-\frac{3}{\hbar^{2}} \hat{\mathbb{L}}_{[2,-1,2]}-\frac{7}{\hbar^{2}} \hat{\mathbb{L}}_{[2,0,1]}-\frac{8}{\hbar^{2}} \hat{\mathbb{L}}_{[1,0,2]}+\frac{1}{\left(2 \pi \alpha^{\prime}\right)^{2}} \\
& \times\left(\frac{72}{\hbar}\left[\hat{L}_{2} \cdot \hat{Q} \cdot \hat{L}_{1}-\hat{L}_{1} \cdot \hat{Q} \cdot \hat{L}_{2}\right]+\hbar a \frac{1}{i \hbar} \hat{\mathbb{L}}_{[2,1]}\right), \\
& s=2: \quad 0=-\frac{2}{\hbar^{2}} \hat{\mathbb{L}}_{[2,-1,1]}-\frac{1}{\hbar^{2}} \hat{\mathbb{L}}_{[1,-1,2]}-\frac{6}{\hbar^{2}} \hat{\mathbb{L}}_{[1,0,1]}+\frac{1}{\left(2 \pi \alpha^{\prime}\right)^{2}} \\
& \times\left(12 i\left\{\hat{Q} \cdot \frac{1}{i \hbar} \hat{\mathbb{L}}_{[2,0]}+\frac{1}{i \hbar} \hat{\mathbb{L}}_{[2,0]} \cdot \hat{Q}\right\}\right. \\
& -48 \hat{L}_{1}^{2}-\frac{192}{\left(2 \pi \alpha^{\prime}\right)^{2}} \hat{Q} \cdot \hat{L}_{2} \cdot \hat{Q}+\hbar b \frac{1}{i \hbar} \hat{\mathbb{L}}_{[2,0]} \\
& \left.-\frac{i \hbar c}{\left(2 \pi \alpha^{\prime}\right)^{2}}\left\{\hat{Q} \cdot \hat{L}_{2}+\hat{L}_{2} \cdot \hat{Q}\right\}-\left(\frac{\hbar}{2 \pi \alpha^{\prime}}\right)^{2} d \hat{L}_{2}\right) \text {, } \\
& s=1: \quad 0=-\frac{1}{\hbar^{2}} \hat{\mathbb{L}}_{[2,-2,1]}-\frac{2}{\hbar^{2}} \hat{\mathbb{L}}_{[1,-2,2]}+\frac{3}{\hbar^{2}} \hat{\mathbb{L}}_{[2,-1,0]}-\frac{3}{\hbar^{2}} \hat{\mathbb{L}}_{[0,-1,2]} \\
& +\frac{6}{\hbar^{2}} \hat{\mathbb{L}}_{[1,-1,1]}+\frac{12}{\hbar^{2}} \hat{\mathbb{L}}_{[1,0,0]} \\
& +\frac{1}{\left.2 \pi \alpha^{\prime}\right)^{2}}\left(\frac{24}{\hbar}\left[\hat{L}_{2} \cdot \hat{Q} \cdot \hat{L}_{-1}-\hat{L}_{-1} \cdot \hat{Q} \cdot \hat{L}_{2}\right]\right. \\
& +\left(\frac{24}{2 \pi \alpha^{\prime}}\right)^{2} \hat{Q} \cdot \hat{L}_{1} \cdot \hat{Q}+\hbar e \frac{1}{i \hbar} \hat{\mathbb{L}}_{[2,-1]}+\hbar f \frac{1}{i \hbar} \hat{\mathbb{L}}_{[1,0]} \\
& \left.-i \frac{\hbar g}{\left(2 \pi \alpha^{\prime}\right)^{2}}\left\{\hat{Q} \cdot \hat{L}_{1}+\hat{L}_{1} \cdot \hat{Q}\right\}-\left(\frac{\hbar}{2 \pi \alpha^{\prime}}\right)^{2} r \hat{L}_{1}\right) \text {, }
\end{aligned}
$$




$$
\begin{aligned}
s=0: \quad 0= & -\frac{1}{\hbar^{2}} \hat{\mathbb{L}}_{[2,-2,0]}+\frac{4}{\hbar^{2}} \hat{\mathbb{L}}_{[1,-1,0]}+\frac{1}{\left(2 \pi \alpha^{\prime}\right)^{2}} \\
& \times\left(\hbar s \frac{1}{i \hbar} \hat{\mathbb{L}}_{[2,-2]}+\hbar t \frac{1}{i \hbar} \hat{\mathbb{L}}_{[1,-1]}-i \frac{\hbar u}{\left(2 \pi \alpha^{\prime}\right)^{4}} \hat{Q}^{3}-i \frac{\hbar^{3} v}{\left(2 \pi \alpha^{\prime}\right)^{4}} \hat{Q}\right) .
\end{aligned}
$$

Next, I pass to the dimensionless operators $\hat{\mathrm{Q}}, \hat{\mathbb{K}}_{0}, \hat{\mathbb{K}}_{s}, s= \pm 1, \pm 2$ :

$$
\begin{aligned}
& s=5: \quad 0=\hat{\mathbb{K}}_{[2,1,2]}, \\
& s=4: \quad 0=9 \hat{\mathbb{K}}_{[2,0,2]}+4 \hat{\mathbb{K}}_{[2,1,1]}-48 \hat{\mathbb{K}}_{2}^{2} \text {, } \\
& s=3: \quad 0=3 \hat{\mathbb{E}}_{[2,-1,2]}+7 \hat{\mathbb{E}}_{[2,0,1]}+8 \hat{\mathbb{A}}_{[1,0,2]} \\
& -72\left[\hat{\mathbb{Z}}_{2} \cdot \hat{\mathbb{Q}} \cdot \hat{\mathbb{Z}}_{1}-\hat{\mathbb{Z}}_{1} \cdot \hat{\mathbb{Q}} \cdot \hat{\mathbb{Z}}_{2}\right]+i a \hat{\mathbb{E}}_{[2,1]} \text {, } \\
& s=2: \quad 0=2 \hat{\mathbb{X}}_{[2,-1,1]}+\hat{\mathbb{E}}_{[1,-1,2]}+6 \hat{\mathbb{A}}_{[1,0,1]}-12\left\{\hat{\mathbb{Q}} \cdot \hat{\mathbb{A}}_{[2,0]}+\hat{\mathbb{A}}_{[2,0]} \cdot \hat{\mathbb{Q}}\right\}
\end{aligned}
$$

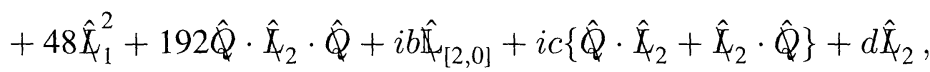

$$
\begin{aligned}
& s=1: \quad 0=\hat{\mathbb{A}}_{[2,-2,1]}+2 \hat{\mathbb{A}}_{[1,-2,2]}-3 \hat{\mathbb{A}}_{[2,-1,0]}+3 \hat{\mathbb{A}}_{[0,-1,2]}-6 \hat{\mathbb{A}}_{[1,-1,1]} \\
& -12 \hat{\mathbb{E}}_{[1,0,0]}-24\left[\hat{\mathbb{A}}_{2} \cdot \hat{\mathbb{Q}} \cdot \hat{\mathbb{A}}_{-1}-\hat{\mathbb{A}}_{-1} \cdot \hat{\mathbb{Q}} \cdot \hat{\mathbb{A}}_{2}\right]-(24)^{2} \hat{\mathbf{Q}} \cdot \hat{\mathbb{A}}_{1} \cdot \hat{\mathbf{Q}} \\
& +i e \hat{\mathbb{K}}_{[2,-1]}+i f \hat{\mathbb{A}}_{[1,0]}+i g\left\{\hat{\mathbb{Q}} \cdot \hat{\mathbb{A}}_{1}+\hat{\mathbb{K}}_{1} \cdot \hat{\mathbb{Q}}\right\}+r \hat{\mathbb{A}}_{1} \text {, } \\
& s=0: \quad 0=\hat{\mathbb{E}}_{[2,-2,0]}-4 \hat{\mathbb{E}}_{[1,-1,0]}+i s \hat{\mathbb{E}}_{[2,-2]}+i t \hat{\mathbb{E}}_{[1,-1]}+i u \hat{\mathbb{Q}}^{3}+i v \hat{\mathbb{Q}} .
\end{aligned}
$$

\section{Conclusions}

In this communication I have formulated and substantiated a program for the quantization of the bosonic closed string moving in $(d-1)+1$-dimensional Minkowski space-time. The program is purely algebraic. Mutatis mutandis, such a program might be applicable also to the sourceless Einstein gravity in 3+1-dimensions - once sufficiently many charges invariant under general coordinate transformations have been identified.

For the actual computations in the string case, resulting in a conjecture about the detailed structure of the algebra of observable symmetries, I have restricted myself to $d=3$. The reason for this restriction was purely technical: the simplicity of the irreducible representations of the stabilizer group $O(2)$ of the momentum rest frame and the relatively small number of non-exceptional generators of the algebra of observable symmetries. A slight irregularity of the structure of the algebra of observable symmetries for the case $d=3$ was the price for the technical simplifications. For the more interesting case: $d=4$, the stabilizer group of the momentum rest frame is $O(3)$ and the set of non-exceptional generators of degree $\ell=1$ consists of the following irreducible representations of $S O(3)$ : two representations with $l=2$ and one representation with $l=1$. For the computation of the generating relations it is helpful to introduce an algebraic basis of $\mathfrak{h} / \hat{\mathfrak{h}}$ consisting of (reparametrization invariant) "irreducible tensor charges." Naturally, the generating relations involve Clebsch-Gordan coefficients. The first characteristic generating relations arise already in the stratum of degree $\ell=2$, i.e. among the simple Poisson 
brackets/the simple commutators. Apparently, the algebra of observable symmetries for the case of positive mass has the following structure:

$$
\mathfrak{s o}(1,3)_{\text {boosts }} \uplus\left\{\mathbb{M}^{4} \oplus\left(\hat{\mathfrak{A}}^{+} \uplus \hat{U}^{+}\right) \oplus\left(\hat{\mathfrak{A}}^{-} \uplus \hat{U}^{-}\right)\right\},
$$

where $\hat{\mathfrak{A}}^{ \pm}$denote infinite dimensional abelian algebras generated by the hermitian scalar elements $\hat{B}_{\ell}^{ \pm}, \ell=1,3,5,7, \ldots$ corresponding to the exceptional elements and where $\hat{U}^{ \pm}$denote algebras generated by the infinitesimal generators of $S O(3)$ and the non-exceptional elements of grade 2 . The superscripts \pm refer to right- and left-movers, respectively.

A host of problems remains open. To mention but the obvious ones, it would be highly desirable to gain insight into the systematics of the generating relations and to prove the above conjectures concerning the structure of the Poisson algebra/commutator algebra of observable symmetries.

\section{Appendix}

The complete expression for $B_{5}$ is:

$$
\begin{aligned}
& B_{5}=(16)^{4} L_{(5)}-(16)^{4} L_{(1)} \cdot L_{(3)}+\frac{1}{3}(16)^{4} L_{(1)}^{3} \\
&+\left[1 6 \left(-\frac{28}{3} \mathbb{L}_{((1,-2,2),(1,-2))}+\frac{70}{3} \mathbb{L}_{((2,-2,1),(1,-2))}+\frac{56}{3} \mathbb{L}_{((2,-2,-1),(1,0))}\right.\right. \\
&-18 \mathbb{L}_{((1,-2,-1),(2,0))}-\frac{32}{3} \mathbb{L}_{((0,-2,-1),(2,1))}-8 \mathbb{L}_{((1,-2,0),(2,-1))} \\
&-\frac{88}{9} \mathbb{L}_{((-1,-2,0),(2,1))}-\frac{224}{3} \mathbb{L}_{((1,-2,2),(0,-1))}-\frac{112}{3} \mathbb{L}_{((1,-1,1),(1,-2))} \\
&-\frac{230}{3} \mathbb{L}_{((1,-2,1),(1,-1))}-\frac{196}{9} \mathbb{L}_{(-1,-2,1,1,1)}+8 \mathbb{L}_{((0,-2,0),(2,0))} \\
&-112 \mathbb{L}_{((1,-2,0),(1,0))}-\frac{418}{3} \mathbb{L}_{(0,-2,0,1,1)}+56 \mathbb{L}_{((1,0,0),(1,-2))} \\
&-60 \mathbb{L}_{((1,0,1),(0,-2))}-140 \mathbb{L}_{((0,-2,1),(1,0))}-\frac{64}{3} \mathbb{L}_{((1,-1,-1),(1,0))} \\
&-128 \mathbb{L}_{(0,-1,-1,1,1)}-208 \mathbb{L}_{((1,-1,0),(1,-1))}-128 \mathbb{L}_{(0,-1,0,0,1)} \\
&\left.+128 \mathbb{L}_{((0,-1,0),(1,0))}\right)-(16)^{2} i Q \cdot\left(-4 \mathbb{L}_{(2,-2,-2,2)}-\frac{100}{9} \mathbb{L}_{((2,1),(-1,-2))}\right. \\
&-11 \mathbb{L}_{(1,-2,0,1)}+\mathbb{L}_{((1,0),(1,-2))}+\frac{511}{9} \mathbb{L}_{(0,-2,1,1)}-\frac{296}{9} \mathbb{L}_{(1,-1,-1,1)} \\
&\left.+65 \mathbb{L}_{(1,-1,0,0)}+40 \mathbb{L}_{((1,0),(0,-1))}\right)+(16)^{2} L_{-2} \cdot\left(-8 \mathbb{L}_{(2,-2,2)}+\frac{65}{3} \mathbb{L}_{(1,-1,2)}\right. \\
&\left.-\frac{356}{3} \mathbb{L}_{(2,0,0)}+\frac{242}{3} \mathbb{L}_{(1,0,1)}\right)+(16)^{2} L_{-1} \cdot\left(-\frac{254}{3} \mathbb{L}_{(1,-2,2)}\right. \\
&\left.+\frac{967}{3} \mathbb{L}_{(2,-1,0)}-\frac{61}{3} \times 16 \mathbb{L}_{(0,-1,2)}+\frac{1172}{3} \mathbb{L}_{(1,-1,1)}+754 \mathbb{L}_{(1,0,0)}\right) \\
&+(16)^{3} Q^{2} \cdot\left(\frac{265}{4} \mathbb{L}_{(0,-2,2)}-\frac{2143}{18} \mathbb{L}_{(1,-2,1)}-119 \mathbb{L}_{(0,-1,1)}\right) \\
&+(16)^{2}\left(-\frac{9}{2} \mathbb{L}_{(-1,-2)} \cdot \mathbb{L}_{(2,1)}+76 \mathbb{L}_{(0,-2)} \mathbb{L}_{(2,0)}+\frac{29}{6} \mathbb{L}_{(1,-2)} \cdot \mathbb{L}_{(2,-1)}\right. \\
&+\frac{701}{3} \mathbb{L}_{(1,-2)} \cdot \mathbb{L}_{(1,0)}+\frac{(16)^{2}}{3} \mathbb{L}_{(0,-1)} \cdot \mathbb{L}_{(1,0)}-\frac{139}{3} \mathbb{L}_{(2,-2)} \cdot \mathbb{L}_{(1,-1)} \\
&\left.+154\left(\mathbb{L}_{(1,-1)}\right)^{2}\right)-(16)^{3} i Q \cdot\left(\frac{911}{18} L_{-2} \cdot \mathbb{L}_{(2,0)}-\frac{191}{2} L_{-1} \cdot \mathbb{L}_{(2,-1)}\right. \\
&\left.-\frac{938}{9} L_{-1} \cdot \mathbb{L}_{(1,0)}+\frac{21560}{9} Q^{2} \cdot \mathbb{L}_{(1,-1)}\right)+(16)^{3}\left(\frac{713}{6} L_{-2} \cdot L_{1}^{2}\right. \\
&\left.\left.-\frac{601}{9} \times 16 Q^{2} \cdot L_{-2} \cdot L_{2}-\frac{152}{9} Q^{2} \cdot L_{-1} \cdot L_{1}-\frac{4616}{9} \times 16 Q^{6}\right)\right] \\
&+\left[L_{s} \leftrightarrow L_{-s}, s=0, \pm 1, \pm 2, Q \leftrightarrow-Q\right] \\
&
\end{aligned}
$$


Acknowledgements. I am indebted to K. Happle for checking the formulae on a computer and for locating my initial numerical errors. I would like to thank the staff of the Physics Department of the University of California at Berkeley for the kind hospitality extended to me during the fall of 1991 and spring of 1992. Financial support by Volkwagenstiftung is gratefully acknowledged.

\section{References}

1. Nambu, J.: Lectures at the Copenhagen Summer Symposium, 1970 (unpublished)

Goto, T.: Relativistic Quantum Mechanics of one-dimensional mechanical continuum and subsidiary condition of dual resonance model. Progr. Theor. Phys. 46, 1560 (1971)

2. Nitsche, J.C.C.: Vorlesungen über Minimalflächen. Die Grundlehren der mathematischen Wissenschaften, Band 199. Berlin, Heidelberg, New York: Springer, 1975

3. Pohlmeyer, K., Rehren, K.-H.: The Invariant Charges of the Nambu-Goto Theory: Their Geometric Origin and Their Completeness. Commun. Math. Phys. 114, 177 (1988)

4. Pohlmeyer, K.: The Invariant Charges of the Nambu-Goto Theory in WKB-Approximation: Renormalization. Commun. Math. Phys. 105, 629 (1986)

5. Lüscher, M., Symanzik, K., Weisz, P.: Anomalies of the free loop wave equation in the WKBApproximation. Nucl. Phys. B 173, 365 (1980)

6. Pohlmeyer, K., Rehren, K.-H.: Algebraic Properties of the Invariant Charges of the Nambu-Goto Theory. Commun. Math. Phys. 105, 593 (1986)

7. Pohlmeyer, K., Rehren, K.-H.: The Algebra Formed by the Invariant Charges of the NambuGoto Theory: Identification of a Maximal Abelian Subalgebra. Commun. Math. Phys. 114, 55 (1988)

8. Pohlmeyer, K.: The Poisson Algebra of the Invariant Charges of the Nambu-Goto Theory: Casimir Elements. Commun. Math. Phys. 114, 351 (1988)

9. Kornhaß, T.: Erhaltungsgrößen auf verzweigten Stringtrajektorien der Nambu-Goto Theorie. Inaugural Dissertation, Fakultät für Physik, Universität Freiburg, 1991

10. Pohlmeyer, K.: The Poisson Algebra Formed by the Invariant Charges of the Nambu-Goto Theory of Closed Strings Moving in 1+2-dimensional Minkowski Space: The Derived Algebra. Preprint THEP 91/1, Fakultät für Physik, Universität Freiburg, 1991

11. Hall, M.A.: A Basis For Free Lie Rings And Higher Commutators In Free Groups. Proc. Am. Math. Soc. 1, 575 (1950)

Communicated by N. Yu. Reshetikhin 
